\title{
Toeplitz matrices and convergence
}

\author{
by
}

Heike Mildenberger (Jerusalem)

\begin{abstract}
We investigate $\left\|\chi_{\mathbb{A}, 2}\right\|$, the minimum cardinality of a subset of $2^{\omega}$ that cannot be made convergent by multiplication with a single matrix taken from $\mathbb{A}$, for different sets $\mathbb{A}$ of Toeplitz matrices, and show that for some sets $\mathbb{A}$ it coincides with the splitting number. We show that there is no Galois-Tukey connection from the chaos relation on the diagonal matrices to the chaos relation on the Toeplitz matrices with the identity on $2^{\omega}$ as first component. With Suslin c.c.c. forcing we show that $\left\|\chi_{\mathbb{M}, 2}\right\|<\mathfrak{b} \cdot \mathfrak{s}$ is consistent relative to ZFC.
\end{abstract}

1. Introduction. A way of generalizing the notion of limit for bounded sequences (i.e. sequences in $\ell^{\infty}$ ) or 0 -1-sequences $\left(2^{\omega}\right)$ is obtained by using the so-called summation methods. These methods work as follows: We fix an $\omega \times \omega$ matrix $A=\left(a_{i, j}\right)_{i, j \in \omega}$ and consider for $f \in \ell^{\infty}$ or $f \in 2^{\omega}$ the product $A \cdot f$ (where, as usual in linear algebra, $\left.(A \cdot f)(i)=\sum_{j \in \omega} a_{i, j} f(j)\right)$. We are interested whether

$$
A \lim f:=\lim _{i \rightarrow \infty} \sum_{j=0}^{\infty}\left(a_{i, j} f(j)\right)
$$

exists.

Below, the quantifier $\forall^{\infty}$ means "for all but finitely many". Toeplitz (cf. [6]) showed: $A \lim$ is an extension of the ordinary limit iff $A$ is a regular matrix, i.e. iff

(i) $(\exists m)(\forall i) \sum_{j=0}^{\infty}\left|a_{i, j}\right|<m$,

(ii) $\lim _{i \rightarrow \infty} \sum_{j=0}^{\infty} a_{i, j}=1$, and

(iii) $(\forall j) \lim _{i \rightarrow \infty} a_{i, j}=0$.

Regular matrices are also called Toeplitz matrices.

2000 Mathematics Subject Classification: Primary 03E35.

The author gratefully acknowledges partial support by a Lise Meitner fellowship of the state of North Rhine Westphalia and by a Minerva fellowship. 
We are going to work with certain subsets of the set $\mathbb{M}$ of all Toeplitz matrices.

Definition 1.1. (a) $\mathbb{L}$ denotes the set of all linear Toeplitz matrices. A matrix is linear iff each column $j$ has at most one entry $a_{i, j} \neq 0$ and for $j<j^{\prime}$ the $i$ with $a_{i, j} \neq 0$ is smaller than or equal to the $i$ with $a_{i, j^{\prime}} \neq 0$ if both exist.

(b) $\mathbb{L}_{\mathrm{u}}$ denotes the set of all linear uniform Toeplitz matrices. A matrix is linear uniform iff there are strictly increasing $c(i), i \in \omega$, such that for each $i$,

$$
a_{i, j}= \begin{cases}\frac{1}{c(i)-c(i-1)} & \text { for } j \in[c(i-1), c(i)), \\ 0 & \text { for other } j .\end{cases}
$$

Here, we stipulate $c(-1)=0$.

(c) $\mathbb{D}_{k}$ denotes the set of all linear Toeplitz matrices that have at most $k$ non-zero entries in each line. We call the matrices in $\mathbb{D}=\mathbb{D}_{1}$ diagonal matrices.

Following Vojtáš [15], we define for $\mathbb{A} \subseteq \mathbb{M}$ limit relations and their dual chaos relations and their norms:

$$
\begin{aligned}
& L_{\infty, \mathbb{A}}=\left\{(f, A) \mid f \in \ell^{\infty} \wedge A \in \mathbb{A} \wedge A \lim f \text { exists }\right\}, \\
& \chi_{\mathbb{A}, \infty}=\left\{(A, f) \mid A \in \mathbb{A} \wedge f \in \ell^{\infty} \wedge A \lim f \text { does not exist }\right\} .
\end{aligned}
$$

For every binary relation $R$ with domain $X$ and range $Y$ such that $(\forall x \in X)$ $(\exists y \in Y) R(x, y)$ we can define

$$
\|R\|=\min \{|\mathcal{Y}| \mid \mathcal{Y} \subseteq Y \wedge(\forall x \in X)(\exists y \in \mathcal{Y}) R(x, y)\} .
$$

For the above relations this reads

$$
\begin{aligned}
& \left\|L_{\infty, \mathbb{A}}\right\|=\min \left\{|\mathcal{A}| \mid \mathcal{A} \subseteq \mathbb{A} \wedge\left(\forall f \in \ell^{\infty}\right)(\exists A \in \mathcal{A}) A \lim f \text { exists }\right\}, \\
& \left\|\chi_{\mathbb{A}, \infty}\right\|=\min \left\{|\mathcal{F}| \mid \mathcal{F} \subseteq \ell^{\infty} \wedge\right. \\
& \quad(\forall A \in \mathbb{A})(\exists f \in \mathcal{F}) A \lim f \text { does not exist }\} .
\end{aligned}
$$

By replacing $\ell^{\infty}$ by $2^{\omega}$, we get the versions $L_{2, \mathbb{A}}$ and $\chi_{\mathbb{A}, 2}$. Families that

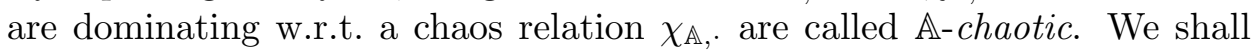
soon see that for the cardinals we are interested in, $2^{\omega}$ and $\ell^{\infty}$ give the same result.

The following inequalities follow from inclusions: $\left\|\chi_{\mathbb{M}, 2}\right\| \geq\left\|\chi_{\mathbb{M}, \infty}\right\| \geq$ $\left\|\chi_{\mathbb{D}, \infty}\right\|$, and $\left\|\chi_{\mathbb{M}, 2}\right\| \geq\left\|\chi_{\mathbb{D}, 2}\right\| \geq\left\|\chi_{\mathbb{D}, \infty}\right\|$. The equality $\left\|\chi_{\mathbb{D}, 2}\right\|=\left\|\chi_{\mathbb{D}, \infty}\right\|=$ $\mathfrak{s}$, the splitting number, is well known [14]. Vojtáš (cf. [16]) also gave some bounds valid for any $\mathbb{A} \supseteq \mathbb{D}$ :

$$
\mathfrak{s} \leq\left\|\chi_{\mathbb{A}, 2}\right\| \leq \mathfrak{b} \cdot \mathfrak{s} .
$$

We shall investigate whether $\left\|\chi_{\mathbb{A}, 2}\right\|$ coincides with the lower or rather with the upper bound in this inequality. 
First we recall the definitions of the cardinal characteristics $\mathfrak{b}$ and $\mathfrak{s}$ involved. The order of eventual dominance $\leq^{*}$ is defined as follows: For $f, g \in \omega^{\omega}$ we say $f \leq^{*} g$ if there is $k \in \omega$ such that for all $n \geq k$ we have $f(n) \leq g(n)$.

The unbounding number $\mathfrak{b}$ is the smallest size of a subset $\mathcal{B} \subseteq \omega^{\omega}$ such that for each $f \in \omega^{\omega}$ there is some $b \in \mathcal{B}$ such that $b \mathbb{Z}^{*} f$. The splitting number $\mathfrak{s}$ is the smallest size of a subset $\mathcal{S} \subseteq[\omega]^{\omega}$ such that for each $X \in[\omega]^{\omega}$ there is some $S \in \mathcal{S}$ such that $X \cap S$ and $X \backslash S$ are both infinite. The latter is expressed as " $S$ splits $X$ ", and $\mathcal{S}$ is called a splitting family. For more information on these cardinal characteristics, we refer the reader to the survey articles $[5,7,13]$.

In [11], Kamburelis and Wȩglorz have introduced a strengthening of splitting, called finitely splitting, FS, and have shown that its norm is fs = $\max (\mathfrak{b}, \mathfrak{s})$. We recall the definition of fs: Let $\mathcal{P}$ be a partition of an infinite subset of $\omega$ into finite sets. A set $X \in[\omega]^{\omega}$ is said to block-split $\mathcal{P}$ if there are infinitely many $P \in \mathcal{P}$ that are included in $X$ and there are infinitely many $P \in \mathcal{P}$ that have empty intersection with $X$. Then we have

$$
\begin{aligned}
\mathrm{FS}= & \left(\text { partitions } \mathcal{P} \text { of an infinite subset of } \omega \text { into finite sets, }[\omega]^{\omega},\right. \\
& \{(\mathcal{P}, X) \mid X \text { block-splits } \mathcal{P}\}), \\
\mathrm{fs}= & \|\mathrm{FS}\| .
\end{aligned}
$$

We give a brief survey of the paper. In Section 2 we show that for any set $\mathbb{A}$ of regular matrices, $\left\|\chi_{\mathbb{A}, \infty}\right\|=\left\|\chi_{\mathbb{A}, 2}\right\|$, so the nature of the bounded sequences (in a separable space) does not have an impact on the chaos numbers. We show that on the matrix side we only need to consider $\mathbb{L}$ and its subsets.

However, we conjecture that the nature of the matrices has an impact. We investigate $\left\|\chi_{\mathbb{A}, 2}\right\|$ for different sets $\mathbb{A}$ of linear Toeplitz matrices.

Section 3 is a technical interlude on block-splitting with bounded block sizes, which is a subrelation of the chaos relation.

In Section 4 we prove: If

$$
\begin{gathered}
(*) \quad(\forall A \in \mathbb{A})(\exists \varepsilon>0)(\exists L \in \omega)\left(\exists^{\infty} i \in \omega\right)\left(\exists j_{0}, \ldots j_{L-1}\right) \\
\sum_{0 \leq l<L} a_{i, j_{l}}>\frac{1}{2}+\varepsilon,
\end{gathered}
$$

then $\left\|\chi_{\mathbb{A}, 2}\right\|=\mathfrak{s}$. Moreover, we prove $\left\|\chi_{\mathbb{L}_{u}, 2}\right\|=\mathfrak{s}$.

In Section 5, we give a model with matrices that shows that it is consistent relative to ZFC that not every splitting family is $\mathbb{L}_{\mathrm{u}}$-chaotic. From the proof we deduce that there is no Galois-Tukey connection from $\chi_{\mathbb{D}, 2}$ to $\chi_{\mathbb{M}, 2}$ with the identity on $2^{\omega}$ as the first component. 
In Section 6, we apply Shelah's theory of Suslin forcing and show that $\left\|\chi_{\mathbb{M}, 2}\right\|<\mathfrak{b} \cdot \mathfrak{s}$ is consistent. I thank Andreas Blass for carefully reading that section.

2. Reduction to 2-valued sequences and to linear matrices. In this section, we show that the range of the bounded sequences can be any separable space; indeed, Vojtášs $\mathfrak{s}=\mathfrak{s}_{\sigma}$ result (cf. [15]) easily generalizes to:

Proposition 2.1. For any $\mathbb{A} \subseteq \mathbb{M}$ such that $\left\|\chi_{\mathbb{A}, \infty}\right\|$ is infinite we have $\left\|\chi_{\mathbb{A}, \infty}\right\|=\left\|\chi_{\mathbb{A}, 2}\right\|$.

We include a proof for completeness' sake and in order that the skeptical reader may see that at this point regular matrices are not harder to treat than diagonal matrices.

Proof. The non-trivial inequality is $\left\|\chi_{\mathbb{A}, \infty}\right\| \geq\left\|\chi_{\mathbb{A}, 2}\right\|$. Without loss of generality, $\ell^{\infty}=[0,1]^{\omega}$. To each $f \in[0,1]^{\omega}$ we assign $\left(g_{f}^{n, k} \mid n \in \omega, k \leq n\right)$, $g_{f}^{n, k} \in 2^{\omega}$, such that

$$
(\forall \text { regular } A)(\forall n, k)\left(A \lim g_{f}^{n, k} \text { exists } \rightarrow A \lim f \text { exists }\right) .
$$

Then we apply this assignment to the set $\mathcal{F}$ witnessing $\left\|\chi_{\mathbb{A}, \infty}\right\|$ to get $\mathcal{F}^{\prime}=$ $\left\{g_{f}^{n, k} \mid n \in \omega, k \leq n, f \in \mathcal{F}\right\}$ witnessing $\left\|\chi_{\mathbb{A}, 2}\right\|$. We set

$$
\begin{aligned}
g_{f}^{n}(j) & = \begin{cases}k & \text { if } f(j) \in[k / n,(k+1) / n), k<n, \\
n-1 & \text { if } f(j) \in[(n-1) / n, 1],\end{cases} \\
g_{f}^{n, k}(j) & = \begin{cases}1 & \text { if } g_{f}^{n}(j)>k, \\
0 & \text { else. }\end{cases}
\end{aligned}
$$

Then $g_{f}^{n}(j)=\sum_{k<n} g_{f}^{n, k}(j)$. It remains to show: If $A \lim f$ does not exist then there are $n$ and $k$ such that $A \lim g_{f}^{n, k}$ does not exist. If the first limit does not exist, then there are two subsequences $\left\langle i_{l, \nu} \mid \nu \in \omega\right\rangle, l=0,1$, such that $\lim _{\nu \rightarrow \infty} \sum_{j} a_{i_{l, \nu}, j} f(j)=: L_{l}$ exist and are distinct. We take $n$ such that $1 / n<\left|L_{0}-L_{1}\right| /(6 m)$, where $m$ is as in property (i) from the introduction.

Then for $l=0,1$ we have

$$
\left(\forall^{\infty} \nu\right)\left|\sum_{j} a_{i_{\ell, \nu}, j}\left(\frac{g_{f}^{n}(j)}{n}-f(j)\right)\right| \leq \frac{L_{0}-L_{1}}{3},
$$

and hence $\lim _{i \rightarrow \infty} \sum_{j} a_{i, j} \sum_{k<n} g_{f}^{n, k}(j)$ does not exist and therefore for one of the summands $\lim _{i \rightarrow \infty} \sum_{j} a_{i, j} g_{f}^{n, k}(j)$ does not exist.

Now we show how to reduce $\mathbb{M}$ to $\mathbb{L}$. This reduction shows that there are Borel morphisms (see [4]) from $\chi_{\mathbb{L}, 2}$ to $\chi_{\mathbb{M}, 2}$ and the same for $\infty$ instead of 2 . 
Proposition 2.2. There is a Borel function $r: \mathbb{M} \rightarrow \mathbb{L}$ such that

$$
\left(\forall f \in \ell^{\infty}\right)(M \lim f \text { exists } \rightarrow r(M) \lim f \text { exists }) .
$$

Proof. Let $M=\left(m_{i, j}\right)_{i, j \in \omega}$ be given. By induction on $n$ we choose line numbers $i(n)$ and indicators $c(n)$ for the edges of the steps of the stairs built by the non-zero entries in $r(\mathbb{M})$. We begin with $i(0)=0$ and $c(0)$ such that

$$
\left|\sum_{j \in \omega} m_{0, j}-\sum_{j<c(0)} m_{0, j}\right| \leq \frac{1}{2} .
$$

Suppose now that $i(n)$ and $c(n)$ have been chosen. Then we choose $i(n+1)>$ $i(n)$ as the first number such that

$$
(\forall j<c(n))\left(\forall i^{\prime} \geq i(n+1)\right)\left|m_{i, j}\right| \leq \frac{1}{c(n) \cdot 2^{n+2}} .
$$

Then we choose $c(n+1)>c(n)$ as the first number such that

$$
\left|\sum_{j \in \omega} m_{i(n+1), j}-\sum_{j<c(n+1)} m_{i(n+1), j}\right| \leq \frac{1}{2^{n+1}} .
$$

Now we set $r(M)=\left(r_{n, j}\right)_{n, j \in \omega}$ with

$$
r_{n, j}= \begin{cases}m_{i(n), j} & \text { if } j \in[c(n), c(n+1)), \\ 0 & \text { else. }\end{cases}
$$

It is routine to check that $r(M)$ is linear Toeplitz and that $r(M) \lim f$ exists whenever $M \lim f$ exists.

So from now on, we work with linear matrices.

3. Finitely splitting with bounded block size. In this section, we investigate some notions of splitting lying between (ordinary) splitting and Kamburelis and Wȩglorz' finitely splitting. We are going to iterate sequential compositions transfinitely often. On sequential compositions, the reader may want to consult [4], yet the knowledge of this background theory is not necessary to understand the proofs given here.

We show that the variant of splitting obtained by taking finitely splitting restricted to sequences $\left\{B_{i} \mid i \in \omega\right\}$ of bounded block size, that is, $(\exists n)(\forall i)\left|B_{i}\right| \leq n$, has $\mathfrak{s}$ as its norm. There are infinitely many sequential compositions involved in our proof. We carry out an induction on a rank, as simple induction on the block size seems to fail.

We fix a splitting family $\mathcal{S}$ and define the first $\omega$ steps in a hierarchy of families with increasing splitting properties yet the same cardinalities ( $S^{\mathrm{c}}$ stands for $\omega \backslash S$ ):

$$
\begin{aligned}
\mathcal{S}^{1} & =\mathcal{S} \cup\{\omega\}, \\
\mathcal{S}^{n+1} & =\left\{(S \cap T) \cup\left(S^{\mathrm{c}} \cap T^{\prime}\right) \mid S \in \mathcal{S}^{1}, T, T^{\prime} \in \mathcal{S}^{n}\right\} .
\end{aligned}
$$


For a sequence $\left\langle X_{i} \mid i<k\right\rangle$ of pairwise disjoint infinite subsets of $\omega$ we say " $S$ splits $\left\langle X_{i} \mid i<k\right\rangle$ simultaneously" if $(\forall i<k)\left|X_{i} \cap S\right|=\left|X_{i} \backslash S\right|=\omega$. The union of the $X_{i}$ need not be $\omega$.

The next lemma contains one idea of the induction step in the more complex splitting situation of Lemma 3.2 and constitutes the first $\omega$ induction steps of the latter lemma.

LEMMA 3.1. For any sequence $\left\langle X_{i} \mid i<k\right\rangle$ of pairwise disjoint infinite subsets of $\omega$ there is some $S \in \mathcal{S}^{k}$ that splits the sequence simultaneously.

Proof. The proof is by induction on $k$. For $k=1$, this is just the ordinary splitting.

$k \rightarrow k+1$ : We first split $\bigcup_{i<k+1} X_{i}$ in the usual sense with some $S \in \mathcal{S}^{1}$.

First case: $S$ already splits every $X_{i}, i<k+1$. As $\mathcal{S} \subseteq \mathcal{S}^{k+1}$, we are finished.

Second case: There are sets $I_{l}, l=0,1,2$, such that

$$
\begin{aligned}
& k+1=I_{0} \cup I_{1} \cup I_{2}, \\
& I_{0} \cup I_{1} \neq \emptyset, \\
& \left(\forall i \in I_{0}\right) X_{i} \subseteq^{*} S, \\
& \left(\forall i \in I_{1}\right) X_{i} \cap S \text { is finite, } \\
& \left(\forall i \in I_{2}\right) S \text { splits } X_{i} .
\end{aligned}
$$

We assume $\left|I_{0} \cup I_{2}\right|<k+1$; otherwise we take $I_{1}$ instead of $I_{0}$. By induction hypothesis, there is some $T \in \mathcal{S}^{k}$ that splits $\left\langle X_{i} \cap S \mid i \in I_{0} \cup I_{2}\right\rangle$ simultaneously and some $T^{\prime} \in \mathcal{S}^{k}$ that splits $\left\langle X_{i} \cap S^{\mathrm{c}} \mid i \in I_{1}\right\rangle$ simultaneously. Then $(S \cap T) \cup\left(S^{\mathrm{c}} \cap T^{\prime}\right)$ splits $\left\langle X_{i} \mid i<k+1\right\rangle$ simultaneously.

For $i \in I_{0}$ we have $X_{i} \subseteq^{*} S$ and $T \cap X_{i}=S \cap T \cap X_{i}$; moreover, $X_{i} \backslash\left(S \cap T \cap X_{i}\right)$ is infinite and $X_{i} \cap S \cap T$ is infinite. Hence also

$$
\begin{aligned}
X_{i} \backslash\left((S \cap T) \cup\left(S^{\mathrm{c}} \cap T^{\prime}\right)\right) & =X_{i} \backslash\left(\left(S \cap T \cap X_{i}\right) \cup\left(S^{\mathrm{c}} \cap T^{\prime}\right)\right) \\
& ={ }^{*} X_{i} \backslash\left(S \cap T \cap X_{i}\right)
\end{aligned}
$$

is infinite.

For $i \in I_{1}$, that $S$ splits $X_{i}$ is shown in a similar manner with $S^{\mathrm{c}}, T^{\prime}$ instead of $S, T$.

For $i \in I_{2}, S \cap X_{i}$ is infinite, and $T \cap X_{i} \cap S$ is infinite, hence $X_{i} \cap$ $\left((S \cap T) \cup\left(S^{\mathrm{c}} \cap T^{\prime}\right)\right)$ is infinite. Moreover, $S \cap X_{i} \backslash T$ is infinite, and hence $S \cap X_{i} \backslash\left((T \cap S) \cup\left(S^{\mathrm{c}} \cap T^{\prime}\right)\right)$ and $X_{i} \backslash\left((T \cap S) \cup\left(S^{\mathrm{c}} \cap T^{\prime}\right)\right)$ are infinite.

The sequential aspect in the sense of [4] is that when choosing $T$ and $T^{\prime}$ we refer to $X_{i} \cap S$ and $X_{i} \cap S^{\mathrm{c}}$. Now we will go beyond $\omega$ and achieve more splitting, going into the direction of finitely splitting from [11]. 
For $\omega$ and for ordinals $\alpha>\omega$ we define

$$
\begin{aligned}
& \mathcal{S}^{\omega}=\bigcup_{n \in \omega} \mathcal{S}^{n}, \\
& \mathcal{S}^{\alpha}=\left\{(S \cap T) \cup\left(S^{\mathrm{c}} \cap T^{\prime}\right) \mid S \in \mathcal{S}^{\omega} \wedge(\exists \beta \in \alpha)\left(T \in \mathcal{S}^{\beta} \wedge T^{\prime} \in \mathcal{S}^{\beta}\right)\right\} .
\end{aligned}
$$

For a sequence $\left\langle\left\{B_{i, j} \mid i \in \omega\right\} \mid j<k\right\rangle$ of sets of pairwise disjoint nonempty finite subsets $B_{i, j}$ of $\omega$ we say " $S$ block-splits $\left\langle\left\{B_{i, j} \mid i \in \omega\right\} \mid j<k\right\rangle$ simultaneously" if $(\forall j<k)\left(\left(\exists^{\infty} i\right)\left(B_{i, j} \subseteq S\right) \wedge\left(\exists^{\infty} i\right)\left(B_{i, j} \cap S=\emptyset\right)\right)$. Thus block-splitting is simultaneous block-splitting for $k=1$.

On the set of all $\left\langle\left\{B_{i, j} \mid i \in \omega\right\} \mid j<k\right\rangle$ such that for all $j<k$ there is $\nu_{j} \in \omega$ such that for all $i$ we have $\left|B_{i, j}\right| \leq \nu_{j}$ we define a rank function $r$ :

$$
r\left(\left\langle\left\{B_{i, j} \mid i \in \omega\right\} \mid j<k\right\rangle\right)=k_{\nu_{0}} \omega^{\nu_{0}-1}+k_{\nu_{1}} \omega^{\nu_{1}-1}+\ldots+k_{\nu_{m-1}} \omega^{\nu_{m-1}-1},
$$

where $m \in \omega, \nu_{0}>\nu_{1}>\ldots>\nu_{m-1}$, and for $\nu \in \omega$,

$$
\begin{aligned}
k_{\nu} & =\left|\left\{j<k \mid(\forall i)\left(\left|B_{i, j}\right| \leq \nu\right) \wedge(\exists i)\left(\left|B_{i, j}\right|=\nu\right)\right\}\right|, \\
k & =k_{\nu_{0}}+k_{\nu_{1}}+\ldots+k_{\nu_{m-1}} .
\end{aligned}
$$

The exponentiation, multiplication, and addition are to be interpreted as ordinal operations in this section.

LEMma 3.2. For all $n, k \in \omega \backslash\{0\}$ for any sequence $\bar{B}=\left\langle\left\{B_{i, j} \mid i \in \omega\right\}\right|$ $j<k\rangle$ of sets of pairwise disjoint non-empty subsets $B_{i, j}$ of $\omega$ such that each $\left|B_{i, j}\right|$ is less than $n$ there is some $S \in \mathcal{S}^{r(\bar{B})}$ that simultaneously block-splits $\left\langle\left\{B_{i, j} \mid i \in \omega\right\} \mid j<k\right\rangle$.

Pr o of. The proof is by induction on the $\operatorname{rank} r(\bar{B})$. For $r(\bar{B})<\omega$, this is Lemma 3.1.

Induction step: Suppose for all $\bar{B}$ with $r(\bar{B})<\alpha$ the claim is proved and $\bar{B}$ with $r(\bar{B})=\alpha \geq \omega$ is given.

First we simultaneously split with some $S \in \mathcal{S}^{k}$ the sequence

$$
\left\langle\bigcup_{i \in \omega} B_{i, j} \mid j \in k\right\rangle .
$$

First case: For all $j<k, S$ block-splits $\left\{B_{i, j} \mid i \in \omega\right\}$. Then $S \in \mathcal{S}^{k} \subseteq$ $\mathcal{S}^{r(\bar{B})}$ is as desired.

Second case: Not the first case. Then there is some $j<k$ such that

$$
\left(\exists^{\infty} i\right)\left(S \cap B_{i, j} \neq \emptyset \wedge S^{\mathrm{c}} \cap B_{i, j} \neq \emptyset\right) .
$$

We define

$$
\begin{aligned}
& J_{0}=\left\{j<k+1 \mid S \text { block-splits }\left\{B_{i, j} \mid i \in \omega\right\}\right\}, \\
& J_{1}=\left\{j<k+1 \mid S \text { does not block-split }\left\{B_{i, j} \mid i \in \omega\right\}\right\},
\end{aligned}
$$


and have $J_{1} \neq \emptyset$. If $j \in J_{1}$, then since $S$ splits $\bigcup\left\{B_{i, j} \mid i \in \omega\right\}$ and does not block-split $\left\{B_{i, j} \mid i \in \omega\right\}$ we see that

$$
L_{j}:=\left\{i \mid S \cap B_{i, j} \neq \emptyset \wedge S^{\mathrm{c}} \cap B_{i, j} \neq \emptyset\right\}
$$

is infinite. We take

$$
\bar{B}^{\prime}=\left\langle\left\{B_{i, j} \mid B_{i, j} \subseteq S, i \in \omega\right\} \mid j \in J_{0}\right\rangle^{\wedge}\left\langle\left\{B_{i, j} \cap S \mid i \in L_{j}\right\} \mid j \in J_{1}\right\rangle .
$$

Since $J_{1} \neq \emptyset$ and since for $j \in J_{1}, i \in L_{j}$ we have $\left|B_{i, j} \cap S\right|<\left|B_{i, j}\right|$, the $\operatorname{rank} \beta$ of $\bar{B}^{\prime}$ is less than $\alpha$. Hence by induction hypothesis there is some $T \in \mathcal{S}^{\beta}$ that simultaneously block-splits $\bar{B}^{\prime}$.

Similarly, by induction hypothesis there is some $\beta^{\prime}<\alpha$ and some $T^{\prime} \in$ $\mathcal{S}^{\beta^{\prime}}$ that simultaneously block-splits

$$
\begin{aligned}
\bar{B}^{\prime \prime}= & \left\langle\left\{B_{i, j} \backslash S \mid i \in L_{j}, B_{i, j} \cap S \subseteq T\right\} \mid j \in J_{1}\right\rangle \\
& \wedge\left\langle\left\{B_{i, j} \backslash S \mid i \in L_{j}, B_{i, j} \cap T \cap S=\emptyset\right\} \mid j \in J_{1}\right\rangle .
\end{aligned}
$$

Now we show that $(S \cap T) \cup\left(S^{\mathrm{C}} \cap T^{\prime}\right)$ simultaneously block-splits $\bar{B}$.

First case: $j \in J_{0}$. Then $X_{j}^{+}=\left\{i \mid B_{i, j} \subseteq S\right\}$ is infinite and $X_{j}^{-}=\{i \mid$ $\left.S \cap B_{i, j}=\emptyset\right\}$ is infinite. Since $T$ block-splits $\left\{B_{i, j} \mid i \in X_{j}^{+}\right\}$, there are infinitely many $i \in X_{j}^{+}$such that $B_{i, j} \subseteq S \cap T$ and there are infinitely many $i \in X_{j}^{+}$such that $B_{i, j} \subseteq S$ and $B_{i, j} \cap T=\emptyset$. For these latter infinitely many $i$ we have $B_{i, j} \cap\left((S \cap T) \cup\left(S^{\mathrm{c}} \cap T^{\prime}\right)\right)=\emptyset$. So altogether we conclude that $(S \cap T) \cup\left(S^{\mathrm{c}} \cap T^{\prime}\right)$ block-splits $\left\{B_{i, j} \mid i \in \omega\right\}$.

Second case: $j \in J_{1}$. Then $L_{j}$ is infinite. We know that $T$ block-splits $\left\{B_{i, j} \cap S \mid i \in L_{j}\right\}$. So $Y_{j}^{+}=\left\{i \in L_{j} \mid T \supseteq B_{i, j} \cap S\right\}$ and $Y_{j}^{-}=\left\{i \in L_{j} \mid\right.$ $\left.B_{i, j} \cap S \cap T=\emptyset\right\}$ are both infinite. Now $T^{\prime}$ block-splits $\left\{B_{i, j} \backslash S \mid i \in L_{j} \cap Y_{j}^{+}\right\}$ and block-splits $\left\{B_{i, j} \backslash S \mid i \in L_{j} \cap Y_{j}^{-}\right\}$. Hence $Z_{j}^{++}=\left\{i \in L_{j} \cap Y_{j}^{+} \mid\right.$ $\left.B_{i, j} \backslash S \subseteq T^{\prime}\right\}$ and $Z_{j}^{--}=\left\{i \in L_{j} \cap Y_{j}^{-} \mid\left(B_{i, j} \backslash S\right) \cap T^{\prime}=\emptyset\right\}$ are both infinite. For $i \in Z_{j}^{++}$we have $B_{i, j} \subseteq(S \cap T) \cup\left(S^{\mathrm{c}} \cap T^{\prime}\right)$ and for $i \in Z_{j}^{--}$we have $B_{i, j} \cap\left((S \cap T) \cup\left(S^{\mathrm{c}} \cap T^{\prime}\right)\right)=\emptyset$. So again we conclude that $(S \cap T) \cup\left(S^{\mathrm{c}} \cap T^{\prime}\right)$ block-splits $\left\{B_{i, j} \mid i \in \omega\right\}$.

Hence we proved:

Theorem 3.3. For all $n \in \omega \backslash\{0\}$ for any set $\left\{B_{i} \mid i \in \omega\right\}$ of pairwise disjoint non-empty subsets $B_{i}$ of $\omega$ such that each $\left|B_{i}\right|$ is less than $n$ there is some $S \in \mathcal{S}^{r\left(\left\langle\left\{B_{i} \mid i \in \omega\right\}\right\rangle\right)}$ that block-splits $\left\{B_{i} \mid i \in \omega\right\}$.

So, since $\mathcal{S}^{r\left(\left\langle\left\{B_{i} \mid i \in \omega\right\}\right\rangle\right)}$ has cardinality $\mathfrak{s}$, the norm of block-splitting with bounded block size coincides with $\mathfrak{s}$. Moreover, if we consider all $\left\{B_{i} \mid i \in \omega\right\}$ such that there is some $n \in \omega$ with $\left|B_{i}\right| \leq n$ for all $i$, then $\mathcal{S}^{\omega^{\omega}}$ contains for each $\left\{B_{i} \mid i \in \omega\right\}$ some $S$ that block-splits it.

4. Splitting is $\mathbb{A}$-chaotic for some $\mathbb{A}$. There are two sorts of matrices for which we can construct chaotic families of the same size as splitting 
families; indeed, we shall start from splitting families and modify them in a very controlled manner. In the first subsection we consider matrices with concentrated weights (which comprise all the $\mathbb{D}_{k}$ ) and make use of the previous section. In the second subsection we modify splitting families in a different manner and get $\mathbb{L}_{\mathrm{u}}$-chaotic families.

Matrices with concentrated weights. Using the results from the previous section, we now prove:

THEOREM 4.1. Let $\mathbb{D} \subseteq \mathbb{A} \subseteq \mathbb{M}$. If $(*)$ (from the introduction) holds for $\mathbb{A}$, then $\left\|\chi_{\mathbb{A}, 2}\right\|=\mathfrak{s}$.

Proof. Let $\omega^{\omega}$ denote the ordinal exponentiation in this proof. We show that $\mathcal{S}^{\omega^{\omega}}$ is a witness for $\left\|\chi_{\mathbb{A}, 2}\right\|=\mathfrak{s}$ for any $\mathbb{A}$ with $(*)$ and that $\mathcal{S}^{\omega^{L}}$ is a witness for $\left\|\chi_{\mathbb{A}, 2}\right\|=\mathfrak{s}$ uniformly for each $\mathbb{A}$ that fulfils (*) uniformly with some fixed $L$. We fix for each $A \in \mathbb{A}$ some $L$ as in $(*)$ and a set $X$ of infinitely many $i$ 's and sets $\left\{j_{0}(i), \ldots, j_{L-1}(i)\right\}$ for $i \in X$ such that

$$
\left\langle\left\{j_{0}(i), \ldots, j_{L-1}(i)\right\} \mid i \in X\right\rangle
$$

is pairwise disjoint. For the disjointness, we use clause (iii) from the definition of a regular matrix. Then we block-split $(k=1$ now $)$ the sequence $\left\{\left\{j_{0}(i), \ldots, j_{L-1}(i)\right\} \mid i \in X\right\}$ by some $S \in \mathcal{S}^{\omega}$. In the following $\chi$ denotes the characteristic function, $\chi_{S}(j)=0$ if $j \notin S$, and $\chi_{S}(j)=1$ else. Then $\lim _{i \in X} \sum_{\nu=1}^{L} a_{i, j_{\nu}(i)} \cdot \chi_{S}\left(j_{\nu}(i)\right)$ does not exist, because the sum is infinitely often zero and infinitely often greater than $1 / 2+\varepsilon$. As the matrix $A$ is regular and hence has properties (i) and (ii), also $\lim _{i \in X} \sum_{j} a_{i, j} \cdot \chi_{S}(j)$ does not exist. Since $\mathfrak{s}$ is infinite, for countable $\alpha$ the cardinality of $\mathcal{S}^{\alpha}$ is $\mathfrak{s}$.

Uniform matrices. With rather basic techniques we show:

THEOREM 4.2. There are Borel functions even, odd : $[\omega]^{\omega} \rightarrow[\omega]^{\omega} \cup$ \{undefined\} and there is a Borel function $f:[\omega]^{\omega} \rightarrow 2^{\omega}$ such that for all splitting families $\mathcal{S}$ the set $\{f(a(S)) \mid a \in\{$ even, odd $\}, S \in \mathcal{S}\}$ is $\mathbb{L}_{\mathrm{u}}$-chaotic. Hence $\left\|\chi_{\mathbb{L}_{\mathrm{u}}, 2}\right\|=\mathfrak{s}$.

P r o of. We let Even denote the set of all even natural numbers and Odd the set of all odd ones. We set

$$
\operatorname{even}(X)=(X \cap \text { Even }) \cup\{x-1 \mid x \in X \cap \text { Even, } x \neq 0\}
$$

if this is infinite and otherwise leave it undefined. Analogously, we set

$$
\operatorname{odd}(X)=(X \cap \text { Odd }) \cup\{x-1 \mid x \in X \cap \text { Odd }\}
$$

if this is infinite and else undefined.

Let $\left\{B_{n} \mid n \in \omega\right\}$ be a set of infinitely many blocks $B_{n}$ such that each $B_{n}$ is of the form $B_{n}=\left\{\min \left(B_{n}\right), \min \left(B_{n}\right)+1\right\}$. The union of the $B_{n}$ need not be $\omega$. 
Claim. If $\mathcal{S}$ is a splitting family then $\{a(S) \mid a \in\{$ even, odd $\}, S \in \mathcal{S}\}$ block-splits $\left\{B_{n} \mid n \in \omega\right\}$.

Proof. Assume that $\left\{\max B_{n} \mid n \in \omega\right\} \cap$ Even is infinite (for the other case, just work with Odd). Take $A \in \mathcal{S}$ such that $A$ splits $\left\{\max B_{n} \mid n \in \omega\right\} \cap$ Even. Then for the infinitely many $n$ with $n \in A \cap\left\{\max B_{n} \mid n \in \omega\right\} \cap$ Even we have even $(A) \supseteq B_{n}$, and for the infinitely many $n$ with $n \in\left(\left\{\max B_{n} \mid\right.\right.$ $n \in \omega\} \cap$ Even $) \backslash A$ we have even $(A) \cap B_{n}=\emptyset$, and the claim is proved.

Now we define

$$
f(S)(n)= \begin{cases}1 & \text { if } k \in S \text { and } n \in\left(3^{k-1}, 3^{k}\right], \\ 0 & \text { else. }\end{cases}
$$

Now we show that $\{f(a(S)) \mid a \in\{$ even, odd $\}, S \in \mathcal{S}\}$ is $\mathbb{L}_{\mathrm{u}}$-chaotic. We take a matrix $M \in \mathbb{L}_{\mathrm{u}}$ which is characterised by $\langle c(n) \mid n \in \omega\rangle$. We denote by $\lceil x\rceil$ the least integer greater than or equal to $x$. By the claim, we may take some $S \in \mathcal{S}$ and some $a \in\{$ even, odd $\}$ such that $a(S)$ block-splits

$$
\left\{\left\{\left\lceil\log _{3}(c(n))\right\rceil-1,\left\lceil\log _{3}(c(n))\right\rceil\right\} \mid n \in \omega\right\} .
$$

Then $M \lim f(a(S))$ does not exist because for those infinitely many $k$ such that $k=\left\lceil\log _{3}(c(n))\right\rceil \in a(S)$ and $k-1 \in a(S)$ we have $M \cdot f(a(S))(n) \geq$ $2 / 3$, whereas for those infinitely many $k$ such that $k=\left\lceil\log _{3}(c(n))\right\rceil \notin a(S)$ and $k-1 \notin a(S)$ we have $M \cdot f(a(S))(n) \leq 1 / 3$.

We needed much less than uniformity: For almost all $n$, the weights $m_{n, j}, j \in[c(n), c(n+1))$, have to add up to more than $1 / 2+\varepsilon$ in the right half (or in the right $(K-1) / K$ part for some $K \in \omega$ ) of $[c(n), c(n+1)$ ).

5. Splitting is not necessarily chaotic. In this section we show the following relative consistency:

THEOREM 5.1. The following is consistent relative to ZFC: There is a splitting family $\mathcal{F}$ and a Toeplitz matrix $M$ such that

\section{$M \lim f$ exists for each $f \in \mathcal{F}$.}

Pr o of. We take any model $V$ of ZFC and add $\aleph_{1}$ random reals simultaneously with the measure algebra $B_{\aleph_{1}}$ on $2^{\aleph_{1}}$. Let $G$ be a generic filter and let $\mathcal{F}=\left\{G_{\alpha} \mid \alpha \in \omega_{1}\right\}$ be $\aleph_{1}$ random reals. We show that $\mathcal{F}$ is as claimed in the theorem. Dow [8, Proposition 2.5] shows that these random reals are a splitting family in $V[G]$. Define in $V$ the set $B$ of real numbers $f \in{ }^{\omega} 2$ such that

$$
\lim _{n \rightarrow \infty} \frac{1}{n} \sum_{i<n} f(i)=\frac{1}{2}
$$

The strong law of large numbers (cf. e.g. [2]) says that $B$ has Lebesgue measure one. Moreover, $B$ is Borel. Random reals are generic for random 
forcing and hence lie in every Borel measure 1 set coded in the ground model. So they lie in $B^{*}$, the evaluation of the Borel code (see $[9, \S 42$, pp. 537, 538]) of $B$ in $V[G]$. We have to show that the statement "for all $x \in B$, the arithmetic mean limit exists" is absolute from $V$ to $V[G]$. A formalization for the statement is:

$$
\begin{aligned}
(\forall x)\left(x \in B \rightarrow(\forall k \in \omega)\left(\exists n_{0} \in \omega\right)\left(\forall m, n \geq n_{0}\right)\right. \\
\left.\qquad\left|\sum_{i<n} \frac{x(i)}{n}-\sum_{i<m} \frac{x(i)}{m}\right|<\frac{1}{k}\right) .
\end{aligned}
$$

This is $\Pi_{1}^{1}$ and hence absolute by Shoenfield's theorem. Since $\mathcal{F} \subseteq B^{*}$, all elements of $\mathcal{F}$ do have arithmetic mean limits and $\mathcal{F}$ is not chaotic.

Theorem 5.1 gives a partial answer to Vojtáš' question in [16] whether there is a Borel Galois-Tukey connection $(E, F)$ from $\chi_{\mathbb{M}, 2}$ into $\chi_{\mathbb{D}, 2}$, i.e. $E, F$ such that

$$
\begin{aligned}
& E: \mathbb{M} \rightarrow \mathbb{D}, \quad F: 2^{\omega} \rightarrow 2^{\omega}, \\
& \begin{aligned}
(\forall M \in \mathbb{M})\left(\forall f \in 2^{\omega}\right)(E(M) \lim f & \text { does not exist } \\
& \rightarrow M \lim F(f) \text { does not exist }) .
\end{aligned}
\end{aligned}
$$

Namely, if $f$ ranges over the above splitting family $\mathcal{F}$ then $F$ cannot be the identity.

Since "There are Borel functions $(E, F)=(E$, id $)$ such that $(5.1)$ holds" is a $\Sigma_{2}^{1}$-statement which is not true in the model from Theorem 5.1 , its negation holds in every model of ZFC.

6. $\chi_{\mathbb{M}, 2}$ and finitely splitting. Vojtáš $[14]$ showed $\chi_{\mathbb{M}, 2} \leq \mathfrak{b} \cdot \mathfrak{s}$. Indeed, it is easy to see that there is a Borel Galois-Tukey connection from $\chi_{\mathbb{M}, 2}$ into Kamburelis and Wegglorz' finitely splitting relation. From the next theorem it follows that there is no Borel Galois-Tukey connection in the opposite direction.

THEOREM 6.1. $\chi_{\mathbb{M}, 2}<\mathfrak{b s}$ is consistent relative to ZFC.

Proof. Let $V$ be a model of $\mathrm{CH}$ and add $\kappa \geq \aleph_{2}$ dominating reals by a finite support iteration of c.c.c. Suslin forcings $\left\langle P_{\alpha}, \dot{Q}_{\beta} \mid \beta<\kappa, \alpha \leq \kappa\right\rangle$ (see [1] or [10]). E.g. we can take Hechler reals, that is, extend successively with $\left(Q_{\alpha}, \leq_{Q_{\alpha}}\right)=\left(Q, \leq_{Q}\right)$ where

$$
\begin{gathered}
Q=\left\{(s, F) \mid s \in \omega^{<\omega}, F \subseteq\left(\omega^{\omega}\right)^{M}, F \text { finite }\right\}, \\
(s, F) \leq_{Q}(t, H):=F \supseteq H, s \supseteq t, \\
\quad(\forall h \in H)(\forall i \in \operatorname{dom}(s) \backslash \operatorname{dom}(t)) s(i) \geq h(i) .
\end{gathered}
$$

Let $G_{\kappa}$ be generic over $V$ for this iterated forcing. 
Since we add a dominating real in each step, we get $\mathfrak{b}=\kappa$ in the final model. By [1, Theorem 3.6.21] (the original proof can be found in [10, §3]), the ground model stays a splitting family in the one-step extension. By the stronger theorems from $[10, \S 3]$ we conclude that the ground model stays a splitting family after a finite support iteration of arbitrary length.

Now we show that the ground model not only stays splitting but stays M-chaotic. The proof is a modification of the above mentioned proof for the splitting relation. It is stronger in the sense that we preserve more than splitting.

So 6.1 will be proved with

THEOREM 6.2. Let $P_{\kappa}$ be a $\kappa$-stage finite support iteration of a c.c.c. Suslin forcing. Then $2^{\omega} \cap V$ stays a chaotic family in $V\left[G_{\kappa}\right]$, where $G_{\kappa}$ is any $P_{\kappa}$-generic filter over $V$.

Proof. We will need three lemmas.

Lemma 6.3. Suppose that $\left\{x_{\alpha} \mid \alpha<\omega_{1}\right\} \in V$ is a family of almost disjoint infinite subsets of $\omega$. Let $\dot{m}$ be a $P_{\kappa}$-name for a Toeplitz matrix such that

$$
\Vdash_{P_{\kappa}}\left(\forall \alpha \in \omega_{1}\right) \dot{m} \cdot \chi_{x_{\alpha}} \text { converges. }
$$

Then there exists an $\alpha<\omega_{1}$ such that $\Vdash_{P_{\kappa}} \lim \left(\dot{m} \cdot \chi_{x_{\alpha}}\right)<1 / 2$.

Proof. For every $\alpha$ choose, if possible, a condition $p_{\alpha} \in P$ such that $p_{\alpha} \Vdash_{P_{\kappa}} \lim \left(\dot{m} \cdot \chi_{x_{\alpha}}\right) \geq 1 / 2$. Since the sets $x_{\alpha}$ are almost disjoint and since $\dot{m}$ is a name for a Toeplitz matrix no $2 b+1$ (the $b$ is a bound from property (i) of being Toeplitz; we can assume that $b$ is a natural number) of the $p_{\alpha}$ can have a common lower bound $q$ : Since the weakest element of our forcing order forces $\dot{m}$ to have the properties (i) and (iii) (from the introduction), and since such a $q$ would force that $\lim \left(\dot{m} \cdot \chi_{x_{\alpha_{0}} \cup \ldots \cup x_{\alpha_{2 b-1}}}\right)>b$, this would be a contradiction to the almost disjointness of the $x_{\alpha}$ 's.

Now since $P_{\kappa}$ is c.c.c. there can only be countably many $p_{\alpha}$, because otherwise we could enumerate the defined ones as $\left\{p_{\alpha} \mid \alpha \in \omega_{1}\right\}$ and thin out this set in $\log _{2}(2 b)+1$ steps as follows:

Suppose at the beginning of a step we have $\aleph_{1}$ conditions $\left\{q_{\alpha} \mid \alpha<\omega_{1}\right\}$. In the first step we take $q_{\alpha}$ to be $p_{\alpha}$. Because of the c.c.c. we can find two compatible ones, and we find a witness, say $r_{0}$, of their compatibility. Then we take away the two compatible elements and find among the rest again two compatible ones and take a witness $r_{1}$. We repeat this procedure in $\omega_{1}$ steps. At the limits we take away all formerly chosen pairs. At the end of each step we rename the resulting $r_{\alpha}, \alpha<\omega_{1}$ to be $p_{\alpha}$ again.

After $\log _{2}(2 b)+1$ steps of this procedure, we have a condition $q_{\alpha}$ that lies under at least $4 b$ members of the set $\left\{p_{\alpha} \mid \alpha \in \omega_{1}\right\}$, which would be a contradiction to the property (i) of being Toeplitz with bound $b$. Hence 
only countably many of the $p_{\alpha}$ can be defined. Any $\alpha$ such that $p_{\alpha}$ is not defined has the required property. $\square_{6.3}$

LEMMA 6.4 (Lemma 3.6 .23 of [1]). Let $M$ be a countable model of a sufficiently rich finite part of ZFC. Then there exists a family $\left\{c_{\alpha} \mid \alpha \in \omega_{1}\right\}$ $\subseteq 2^{\omega}$ such that

(i) $c_{\alpha}$ is Cohen over $M$ for every $\alpha<\omega_{1}$ and

(ii) $\left|c_{\alpha}^{-1 \prime \prime}\{1\} \cap c_{\beta}^{-1 \prime \prime}\{1\}\right|<\aleph_{0}$ for $\alpha \neq \beta$.

Proof. See [1, 3.6.23, page 176] or $\S 3$ in [10].

The following lemma finishes the proof of Theorem 6.2:

Lemma 6.5. There is no $\dot{m} \in V^{P_{\kappa}}$ such that

$$
\Vdash_{P_{\kappa}} \dot{m} \text { is Toeplitz, and }
$$

$$
\left(\forall y \in 2^{\omega} \cap V\right) \Vdash_{P_{\kappa}} \dot{m} \cdot y \text { converges. }
$$

Proof. Suppose not. Let $\dot{m}$ be a name for a Toeplitz matrix such that for every $y \in 2^{\omega} \cap V$,

$$
\Vdash_{P_{\kappa}} \dot{m} \cdot y \text { converges. }
$$

Let $S \subseteq \kappa$ be countable and such that $\dot{m} \in V^{P \uparrow S}$. Since $P \uparrow S=P_{S} \lessdot P_{\kappa}$ $([10, \S 1])$ also for every $y \in 2^{\omega} \cap V$,

$$
\Vdash_{P_{S}} \dot{m} \cdot y \text { converges. }
$$

Now we work in $V$. Let $M$ be a countable elementary submodel of $H(\chi)$ for some sufficiently large $\chi$ containing $P_{S}$ and $\dot{m}$. By 6.4, there exists a family $\left\{c_{\alpha} \mid \alpha<\omega_{1}\right\} \subset 2^{\omega}$ of almost disjoint (in the sense of 6.4(ii)) Cohen reals over $M$. By 6.3, we can find a Cohen real $c$ over $M$ such that $\Vdash_{P_{S}} \lim (\dot{m} \cdot c)<1 / 2$. Let $M_{1}=M[c]$. Let $G$ be a $P_{S}$-generic filter over $V$. By $[1,3.6 .5], G \cap M_{1}$ is $P_{S^{-}}$generic over $M_{1}$. Since $\dot{m} \in M$ we have $\dot{m}[G]=\dot{m}\left[G \cap M_{1}\right]=\dot{m}[G \cap M]$. Let $M_{2}=M_{1}\left[G \cap M_{1}\right]$. It follows that

$$
M_{2}=\lim \left(\dot{m}\left[G \cap M_{1}\right] \cdot c\right)<1 / 2 .
$$

Therefore, $M_{1}=" \Vdash_{P_{S}} \lim (\dot{m} \cdot c)<1 / 2$." By the properties of Cohen forcing, which we interpret as $C=\left(2^{<\omega}, \subseteq\right)$, there is some $k \in \omega$ such that $s=c \uparrow k$ and

$$
M \models " s \Vdash_{C} " \Vdash_{P_{S}} \lim (\dot{m} \cdot \dot{c})<1 / 2, " \text { " }
$$

where $\dot{c}$ is a canonical name for a Cohen real.

Let $c^{\prime}$ be such that $s \subseteq c^{\prime}$ and $\left|\omega \backslash\left(c^{-1 \prime \prime}\{1\} \cup\left(c^{\prime}\right)^{-1 \prime \prime}\{1\}\right)\right|<\aleph_{0}$ and $c^{\prime}$ is Cohen real over $M$ and $c^{\prime}$ and $c$ are almost disjoint. So, $M\left[c^{\prime}\right] \models$ "IF $P_{S} \lim \left(\dot{m} \cdot c^{\prime}\right)<1 / 2$ ". Note that $G \cap M\left[c^{\prime}\right]$ is $P_{S^{-}}$generic over $M\left[c^{\prime}\right]$ and $\dot{m}\left[G \cap M\left[c^{\prime}\right]\right]=\dot{m}[G]$. Thus, $M\left[c^{\prime}\right]\left[G \cap M\left[c^{\prime}\right]\right]=\lim \left(\dot{m}[G] \cdot c^{\prime}\right)<1 / 2$. Therefore, in $V[G]$ we have 
1. $\lim (\dot{m}[G] \cdot c)<1 / 2$, and

2. $\lim \left(\dot{m}[G] \cdot c^{\prime}\right)<1 / 2$, and

3. $\left|\omega \backslash\left(c^{-1 \prime \prime}\{1\} \cup\left(c^{\prime}\right)^{-1 \prime \prime}\{1\}\right)\right|<\aleph_{0}$.

This contradicts the fact that $\dot{m}$ was a name for a Toeplitz matrix: Since $c$ and $c^{\prime}$ are almost disjoint, $c+c^{\prime}$ is almost in $2^{\omega}$ and because of property (iii) of Toeplitz matrices we may assume that it is actually in $2^{\omega}$. Then by property (ii) of Toeplitz matrices we have

$$
\lim \left(\dot{m}[G] \cdot\left(c+c^{\prime}\right)\right)=\lim (\dot{m}[G] \cdot c)+\lim \left(\dot{m}[G] \cdot c^{\prime}\right)<1 / 2+1 / 2 .
$$

However, property (i) and 3. imply that

$$
\lim \left(\dot{m}[G] \cdot\left(c+c^{\prime}\right)\right)=1 .
$$

This is a contradiction.

Note that the whole proof of 6.2 would also work with $\lim \leq 1 / 3+\varepsilon$ (for any $\varepsilon<1 / 6)$ instead of $1 / 2$ and limits replaced by eventual ranges of width less than $1 / 6-\varepsilon=: \delta$. So indeed, we have much stronger chaos. $\square_{6.5,6.2,6.1}$

We conjecture $\chi_{\mathbb{M}, 2}$ does not coincide with the splitting number. Finite support iterations of c.c.c. Suslin forcings cannot establish this.

There might be some other finite support iteration or a countable support iteration (of proper forcings [12]) of length $\aleph_{2}$ such that in each iteration step a matrix $M_{\alpha}$ is added and for all $f \in 2^{\omega} \cap V\left[G_{\alpha}\right]$ the $\operatorname{limit} M \lim f$ exists.

REMARK. Being smaller than the linear forgetful evasion number $\mathfrak{e}_{f l}$ (see [3]) seems to be closely related to being non-chaotic: Let $\mathcal{F} \subseteq 2^{\omega}$ be smaller than the linear forgetful evasion number $\mathfrak{e}_{f l}$. Then there is some matrix $M$ obeying ((i) or (ii)) and (iii) of the regularity conditions such that for all members of the family $\mathcal{F}$ we have $M \cdot f=\langle 0,0, \ldots\rangle$. This is proved as in [3]. Theorem 6.2 shows that any finite support iteration of c.c.c. Suslin forcings adding dominating reals and increasing the additivity of Lebesgue null sets add $(\mathcal{N})$ (see [10]) over a ground model with $\mathrm{CH}$ gives a model of $\mathfrak{s}=\chi_{\mathbb{M}, 2}=\aleph_{1}<\kappa=\operatorname{add}(\mathcal{N})=\mathfrak{b}=\min (\mathfrak{e}, \mathfrak{b})=\mathfrak{e}_{f l}$.

\section{References}

[1] T. Bartoszyński and H. Judah, Set Theory. On the Structure of the Real Line, A. K. Peters, Wellesley, MA, 1995.

[2] H. Bauer, Probability Theory, de Gruyter, 4th ed., 1996.

[3] A. Blass, Cardinal characteristics and the product of countably many infinite cyclic groups, J. Algebra 169 (1995), 512-540.

[4] -, Reductions between cardinal characteristics of the continuum, in: T. Bartoszyński and M. Scheepers (eds.), Set Theory (Boise 1992-94), Contemp. Math. 192, Amer. Math. Soc., 1996, 31-49. 
[5] A. Blass, Combinatorial cardinal characteristics of the continuum, in: M. Foreman, A. Kanamori, and M. Magidor (eds.), Handbook of Set Theory, Kluwer, to appear.

[6] R. Cooke, Infinite Matrices and Sequence Spaces, MacMillan, 1950.

[7] E. van Douwen, The integers and topology, in: K. Kunen and J. Vaughan (eds.), Handbook of Set-Theoretic Topology, North-Holland, 1984, 111-167.

[8] A. Dow, Tree $\pi$-bases for $\beta \mathbb{N}-\mathbb{N}$, Topology Appl. 33 (1989), 3-19.

[9] T. Jech, Set Theory, Addison-Wesley, 1978.

[10] H. Judah and S. Shelah, Suslin forcing, J. Symbolic Logic 53 (1988), 1188-1207.

[11] A. Kamburelis and B. Wȩglorz, Splittings, Arch. Math. Logic 35 (1996), 263277.

[12] S. Shelah, Proper Forcing, Lecture Notes in Math. 940, Springer, 1982.

[13] J. E. Vaughan, Small uncountable cardinals and topology, in: J. van Mill and G. Reed (eds.), Open Problems in Topology, Elsevier, 1990, 195-218.

[14] P. Vojtáš, Set theoretic characteristics of summability and convergence of series, Comment. Math. Univ. Carolin. 28 (1987), 173-184.

[15] - , More on set theoretic characteristics of summability by regular (Toeplitz) matrices, ibid. 29 (1988), 97-102.

[16] —, Series and Toeplitz matrices (a global implicit approach), Tatra Mt. Math. J., to appear.

Institute of Mathematics

Hebrew University of Jerusalem

Givat Ram, Jerusalem 91904, Israel

E-mail: heike@math.huji.ac.il

Received 5 July 1999;

in revised form 27 February 2000 